\title{
Finite-Time Adaptive Synchronisation of a Class of Master-Slave Systems with Different Unknown Parameters
}

\author{
Nawel Khelil ${ }^{a}$ and Martin J.-D. Otis ${ }^{b}$ \\ ${ }^{a}$ Polytechnical School Of Tunisia, La Marsa, 2078, Tunis, Tunisia; ${ }^{b}$ REPARTI \\ Center, UQAC, Department of Applied Sciences, Chicoutimi, Quebec, Canada
}

\begin{abstract}
This paper is concerned with the finite-time chaotic synchronization and dynamic errors in finite-time stabilization of master-slave systems. We suggest solving these issues using a virtual recursive adaptive nonlinear controller when different unknown parameters occur. Therefore, a systematic design approach is defined for constructing both virtual adaptive nonlinear feedback control laws and associated Lyapunov functions. The corresponding sufficient conditions to achieve synchronization between two chaotic systems are obtained based on the Lyapunov stability theory. Then, two applications are evaluated using our approach: Genesio-Tesi and Coullet systems which are two topologically dissimilar systems, known as difficult to synchronize. The results presented in this paper demonstrate both effectiveness and feasibility of our control laws.
\end{abstract}

Keywords: chaotic synchronization; finite-time adaptive stabilization; master-slave systems; Coullet system; Genesio-Tesi system

\section{Introduction}

The definition of synchronization of two chaotic systems is in general, the ability of identifying tendency of two or more systems coupled together to undergo closely related motions. Applying this universal concept to dynamical systems, chaos synchronization approach becomes a way to design a coupling interface between both systems such that chaotic time evaluation becomes ideal. In other words, slave system response asymptotically follows the master response (drive system). Therefore, the drive system output controls response of the overall system.

The synchronization of chaotic systems was defined in the earlier works by Fujisaka \& Yamada [9] subsequently a mathematical improvement was given by Carroll \& Pecora [6, 25]. These works shows that despite sensitive dependence on initial conditions, coupled chaotic systems could synchronize. The chaos synchronization has been classified into two types called mutual synchronization and master-slave synchronization according to the coupling configuration defined in [7, 21, 28]. Since the past decades, the problem of synchronization has attracted the attention of the research community area. This problem has been studied due to its potential applications in secure communication [19, 24], in process control [20], in chemical and biological systems [10, 11, 22]. Also, master-slave synchronization has been applied to robotics [27] and even in complex networks [31].

Until now, various effective methods have been developed to synchronize various chaotic systems such as adaptive and impulsive method [33, 36], active control [4], adaptive design method [18, 23], time delay feedback method [24], sliding mode control method [37] and intermittent control [1, 39], amongst others methods.

Most of the methods mentioned above are used to guarantee the asymptotic stability of chaotic systems. In other words, convergence of synchronization process is asymptotic within a finite settling time. However, in the view of practical application, optimizing synchronization time is more important than achieving synchronization asymptotically $[5$, 
15, 36]. Besides, finite-time chaos synchronization control techniques have demonstrated better robustness, better rejection to parameter sensitivities or external disturbances and reduced controller complexity. So far, less attention, in the aforementioned works, has been paid to the issue of finite-time synchronization of chaotic systems with unknown and different parameters.

Motivated by the above discussion, the main contribution of this paper is to propose a new approach in order to design a virtual adaptive controller able to synchronize two topologically dissimilar systems and two other identical chaotic systems. These systems are analyzed with different unknown parameters in finite-time and are defined by classical properties of the finite-time stabilization and Lyapunov stability theory of the corresponding dynamic errors of the system. The main challenge is to design controllers and parameters update laws such that they contain fully unknown parameters, which cannot be implemented in practice. In order to overcome this difficulty, a virtual adaptive controller is designed systemically at each step of the algorithm.

The rest of the paper is organized as follows. In order to explain the contribution, second section introduces the system description, primary definitions and lemma about finite-time chaos synchronization. Then, in the third section, based on the Lyapunov stability theory and our Lemma, an algorithm is realized in order to design our new finite-time synchronizing controller. Following this, numerical simulations are presented in Section 4, with two $3 D$ chaotic systems in two different cases: Genesio-Tesi system and Coullet system. In order to demonstrate effectiveness of the controller, different unknown parameters are used which increase the difficulty. Finally, a discussion and a conclusion of our results is presented.

\section{Proposed System and Finite-Time Chaotic Synchronisation Preliminaries}

In this section, we consider a class of n-dimensional nonlinear master-slave systems. First, the master system is described by the following set of equations:

$$
\left\{\begin{array}{l}
\dot{x}_{1}=x_{2}+f_{1}\left(x_{1}, \theta\right) \\
\dot{x}_{2}=x_{3}+f_{2}\left(x_{1}, x_{2}, \theta\right) \\
\vdots \\
\dot{x}_{n}=f_{n}\left(x_{1}, \cdots, x_{n}, \theta\right) .
\end{array}\right.
$$

Second, concerning the slave system, the following equations are used:

$$
\left\{\begin{array}{l}
\dot{y}_{1}=y_{2}+f_{1}\left(y_{1}, \eta\right) \\
\dot{y}_{2}=y_{3}+f_{2}\left(y_{1}, y_{2}, \eta\right) \\
\vdots \\
\dot{y}_{n}=f_{n}\left(y_{1}, \cdots, y_{n}, \eta\right)+u
\end{array}\right.
$$

where $x(t)=\left(x_{1}, x_{2}, \cdots, x_{n}\right)^{T} \in \mathbb{R}^{n}$ is the state vector of the master system, $f(x)=$ $\left(f_{1}(x), \cdots, f_{n}(x)\right)^{T}$, such that $f_{i}(x)=f_{i}\left(x_{1}, \cdots, x_{i}\right)$, are $C^{1}$ nonlinear functions vanishing at the origin, $\theta=\left(\theta_{1}, \theta_{2}, \cdots, \theta_{n}\right)^{T} \in \mathbb{R}^{n \times 1}$ is an unknown parameter vector of the master system, $y(t)=\left(y_{1}, y_{2}, \cdots, y_{n}\right)^{T} \in \mathbb{R}^{n}$ is the state vector of the slave system, $\eta=$ $\left(\eta_{1}, \eta_{2}, \cdots, \eta_{n}\right)^{T} \in \mathbb{R}^{n \times 1}$ is an unknown parameter vector of the slave system and $u(t) \in \mathbb{R}$ is the control input.

We define the error between master and slave systems using $e(t)=y(t)-x(t)$. Therefore, by subtracting equation (2) from equation (1), one can obtain the following error: 


$$
\left\{\begin{array}{l}
\dot{e}_{1}(t)=e_{2}(t)+f_{1}\left(y_{1}, \eta\right)-f_{1}\left(x_{1}, \theta\right) \\
\dot{e}_{2}(t)=e_{3}(t)+f_{2}\left(y_{1}, y_{2}, \eta\right)-f_{2}\left(x_{1}, x_{2}, \theta\right) \\
\vdots \\
\dot{e}_{n}(t)=u+f_{n}(y, \eta)-f_{n}(x, \theta) .
\end{array}\right.
$$

Finite-time chaos synchronization between both systems (1) and (2) can be addressed as a stabilization problem of dynamic errors (3) in finite-time [2, 3]. This means that the trajectories of the synchronization error have to be stabilized at the origin, i.e. the state of the slave system can track the state of the master system after a finite-time [15, 29, 38].

The precise definition of finite-time synchronization, applied to our case of study, is given below [16]:

Definition 1 Consider the master and slave systems described by equations (1) and (2). If there exists a constant $T=T(e(0))>0$, such that

$$
\lim _{t \rightarrow T}\|e(t)\|=0
$$

and $\|e(t)\| \equiv 0$, if $t \geq T$, then the chaos synchronization between systems (1) and (2) is achieved in a finite-time.

Next, we give a lemma that will be used in the sequel.

Lemma 1: Assume that a continuous, positive-definite Lyapunov function $V(t)$ satisfies the following differential inequality:

$$
\dot{V}(t) \leq-\lambda V^{\eta}(t), \quad \forall t \geq t_{0}, V\left(t_{0}\right) \geq 0,
$$

where $\lambda>0,0<\eta<1$ are both constants. Then, for any given $t_{0}, V(t)$ satisfies the following inequality:

and

$$
V^{1-\eta}(t) \leq V^{1-\eta}\left(t_{0}\right)-\lambda(1-\eta)\left(t-t_{0}\right), \quad \forall t_{0} \leq t \leq t_{1},
$$

$$
V(t)=0, \quad \forall t \geq t_{1},
$$

with $t_{1}=t_{0}+\frac{V^{1-\eta}\left(t_{0}\right)}{\lambda(1-\eta)}$. Therefore, the system can achieve global stability in finite-time.

In the next section, we will give the algorithm steps, under appropriate conditions, to construct our adaptive finite-time controller to guarantee finite-time chaos synchronization between both systems proposed by (1) and (2).

\section{Finite-Time Controller Design and Stability Analysis}

In this section, we investigate the design of a finite-time controller to realize chaos synchronization in a given finite-time between two different or similar n-dimensional chaotic systems (1) and (2) with unknown parameters. Moreover, this virtual finite-time controller assures asymptotic stability in finite-time.

We state conditions in order to provide a recursive systematic algorithm starting from dynamic errors and using the Lyapunov stability theory applied on a step by step resized and reconstructed subsystems. Before going further, we need to consider dynamic errors (3) and then we now introduce these three following assumptions.

(A1) The unknown vector parameters $\theta$ and $\eta$ are norm bounded such that

$$
\|\theta\|_{\Gamma_{\theta}^{-1}}^{2}=\theta^{T} \Gamma_{\theta}^{-1} \theta \leq M_{\theta} \quad,\|\eta\|_{\Gamma_{\eta}^{-1}}^{2}=\eta^{T} \Gamma_{\eta}^{-1} \eta \leq M_{\eta}
$$

where $M_{\theta}$ and $M_{\eta}$ are both known positive constants.

(A2) The unknown function $f(.,$.$) satisfies$

$$
\left\|f_{i}(y, \eta)-f_{i}(x, \theta)\right\| \leq\left\|(x-y)_{i}\right\| h_{i}^{2}(x, y)\|\theta\|_{\Gamma_{\theta}^{-1}}^{2}\|\eta\|_{\Gamma_{\eta}^{-1}}^{2},
$$

where $h_{i}(.,):.\left(\mathbb{R}^{i}\right)^{2} \rightarrow \mathbb{R}^{+}$is a known smooth scaling function satisfying $h_{i}(0,0)=0$ and $(x-y)_{i}=\left(x_{1}-y_{1}, \cdots, x_{i}-y_{i}\right)$ and $\|$.$\| is the Euclidean norm in \mathbb{R}^{n}$.

(A3) The unknown possible nonlinear function $f_{i}(.,$.$) is linearly parametrized, in$ terms of the unknown parameters $\theta_{i}$ and $\eta_{i}$, and that there exist, respectively, two bounded bases $\phi_{i}(x)$ and $\delta_{i}(y)$, such that: 
and

$$
\begin{gathered}
\mathrm{f}_{\mathrm{i}}(\mathrm{x}, \theta)=\mathrm{f}_{\mathrm{i}}\left(\mathrm{x}_{1}, \mathrm{x}_{2}, \cdots, \mathrm{x}_{\mathrm{i}}, \theta\right)=\sum_{\mathrm{j}=1}^{\mathrm{n}} \theta_{\mathrm{j}} \phi_{\mathrm{j}}\left(\mathrm{x}_{1}, \mathrm{x}_{2}, \cdots, \mathrm{x}_{\mathrm{i}}\right) \\
=\theta^{\mathrm{T}} \Phi_{\mathrm{i}}\left(\mathrm{x}_{1}, \mathrm{x}_{2}, \cdots, \mathrm{x}_{\mathrm{i}}\right)=\theta^{\mathrm{T}} \Phi_{\mathrm{i}}(\mathrm{x})
\end{gathered}
$$

$$
\begin{gathered}
f_{i}(y, \eta)=f_{i}\left(y_{1}, y_{2}, \cdots, y_{i}, \eta\right)=\sum_{j=1}^{n} \eta_{j} \delta_{j}\left(y_{1}, y_{2}, \cdots, y_{i}\right) \\
=\eta^{T} \Delta_{i}\left(y_{1}, y_{2}, \cdots, y_{i}\right)=\eta^{T} \Delta_{i}(y) .
\end{gathered}
$$

To proceed further, our aim is to design a constructive controller, such that the two systems (1) and (2) are finite-time synchronized. Then, the problem is changed to design a suitable controller, leading to the error system (3) to achieve the finite-time stability at the origin. The design plan and its steps are described in the following.

Step 1: We choose a positive definite Lyapunov function in the form of

$$
V_{1}\left(e_{1}\right)=\frac{1}{2} e_{1}^{2}+\int_{t_{0}}^{t} e_{1}^{2}(\tau) h_{1}^{2}\left(x_{1}(\tau), y_{1}(\tau)\right) d \tau+\frac{1}{2} \tilde{\theta}^{T} \Gamma_{\theta}^{-1} \tilde{\theta}+\frac{1}{2} \tilde{\eta}^{T} \Gamma_{\eta}^{-1} \tilde{\eta} .
$$

where $\Gamma_{\theta}=\Gamma_{\theta}^{T}>0, \Gamma_{\eta}=\Gamma_{\eta}^{T}>0$ are rates of adaptation and $\tilde{\theta}=\theta-\hat{\theta}, \tilde{\eta}=\eta-\hat{\eta}$ with $\hat{\theta}, \hat{\eta}$ are the esimate values of the unknown parameters $\theta, \eta$ respectively (It is clear that $\dot{\tilde{\theta}}=\dot{\hat{\theta}}$ and $\dot{\tilde{\eta}}=\dot{\hat{\eta}}$ ).

The time derivative of $V\left(e_{1}\right)$ is given by:

$$
\begin{aligned}
\dot{V}_{1}\left(e_{1}\right)= & e_{1} e_{2}+e_{1}\left[f_{1}\left(y_{1}, \eta\right)-f_{1}\left(x_{1}, \theta\right)\right] \\
& +e_{1}^{2} h_{1}^{2}\left(x_{1}, y_{1}\right)+\tilde{\theta}^{T} \Gamma_{\theta}^{-1} \dot{\hat{\theta}}+\tilde{\eta}^{T} \Gamma_{\eta}^{-1} \dot{\hat{\eta}} .
\end{aligned}
$$

The online estimated quantities of $f(x, \theta)$ and $f(y, \eta)$ are respectively:

$\hat{f}_{i}(x, \theta)=\sum_{j=1}^{n} \hat{\theta}_{j} \phi_{j}(x)=\hat{\theta}^{T} \Phi_{i}(x)$

and

$\hat{f}_{i}(y, \eta)=\sum_{j=1}^{n} \hat{\eta}_{j} \delta_{j}(y)=\hat{\eta}^{T} \Delta_{i}(y)$.

Then, the associated functions approximation error can be written as follow:

$\tilde{f}_{i}(x, \theta)=\hat{f}_{i}(x, \theta)-f_{i}(x, \theta)=\sum_{j=1}^{n}\left(\hat{\theta}_{j}-\theta_{j}\right) \phi_{j}(x)=\tilde{\theta}^{T} \Phi_{i}(x)$

and

$\tilde{f}_{i}(y, \eta)=\hat{f}_{i}(y, \eta)-f_{i}(y, \eta)=\sum_{j=1}^{n}\left(\hat{\eta}_{j}-\eta_{j}\right) \delta_{j}(y)=\tilde{\eta}^{T} \Delta_{i}(y)$.

Using (15) and (16) and noting the Assumption (A2), we obtain for (13):

$$
\begin{gathered}
\dot{V}_{1}\left(e_{1}\right)=e_{1}\left(e_{2}-e_{2}^{*}\right)+e_{1} e_{2}^{*}+e_{1}\left[\eta^{T} \Delta_{1}\left(y_{1}\right)-\theta^{T} \Phi_{1}\left(x_{1}\right)\right] \\
+e_{1}^{2} h_{1}^{2}\left(x_{1}, y_{1}\right)+\tilde{\theta}^{T} \Gamma_{\theta}^{-1} \hat{\hat{\theta}}+\tilde{\eta}^{T} \Gamma_{\eta}^{-1} \dot{\hat{\eta}} .
\end{gathered}
$$

By substituting the latter into equation (17), the virtual adaptive error controller is obtained:

$$
e_{2}^{*}=\hat{\theta}^{T} \Phi_{1}\left(x_{1}\right)-\hat{\eta}^{T} \Delta_{1}\left(y_{1}\right)-e_{1} h_{1}^{2}\left(x_{1}, y_{1}\right)-c_{1} e_{1} \text {, (18) }
$$

where $c_{1}>0$ is a constant design parameter. One can hold the inequality:

$\dot{V}_{1}\left(e_{1}\right) \leq e_{1}\left(e_{2}-e_{2}^{*}\right)+\tilde{\theta}^{T}\left[\Gamma_{\theta}^{-1} \dot{\hat{\theta}}+\Phi_{1}\left(x_{1}\right) e_{1}\right]+\tilde{\eta}^{T}\left[\Gamma_{\eta}^{-1} \dot{\hat{\eta}}-\Delta_{1}\left(y_{1}\right) e_{1}\right]-c_{1} e_{1}^{2}$.

Based on (19), in order to make $\dot{V}_{1}\left(e_{1}\right) 0$, the adaptive laws are chosen as:

$\dot{\hat{\theta}}=-\Gamma_{\theta} \Phi_{1}\left(x_{1}\right) e_{1}$ and

$\dot{\hat{\eta}}=\Gamma_{\eta} \Delta_{1}\left(y_{1}\right) e_{1}$.

In fact, this leads to

$\dot{V}_{1}\left(e_{1}\right) \leq e_{1}\left(e_{2}-e_{2}^{*}\right)-c_{1} e_{1}^{2}$.

The function $e_{2}^{*}$ is estimative when $e_{2}$ is considered as a controller. We introduce the following error between $e_{2}$ and $e_{2}^{*}$ as

$w_{2}=e_{2}-e_{2}^{*}$.

Step 2: Consider $\left(e_{1}, w_{2}\right)$ as a subsystem given by: 
$\left\{\begin{array}{l}\dot{e}_{1}(t)=e_{2}(t)+f_{1}\left(y_{1}, \eta\right)-f_{1}\left(x_{1}, \theta\right), \\ \dot{w}_{2}(t)=e_{3}(t)+f_{2}\left(y_{1}, y_{2}, \eta\right)-f_{2}\left(x_{1}, x_{2}, \theta\right)-\dot{e}_{2}^{*} .\end{array}\right.$

We define the Lyapunov function given by

$V_{2}\left(e_{1}, w_{2}\right)=\frac{1}{2} e_{2}^{2}+V_{1}\left(e_{1}\right)+\int_{t_{0}}^{t} e_{2}^{2}(\tau) h_{2}^{2}\left((x, y)_{2}\right)(\tau) d \tau$,

where $(x(\tau), y(\tau))_{2}=\left(x_{1}(\tau), x_{2}(\tau), y_{1}(\tau), y_{2}(\tau)\right)$. Let $e_{3}^{*}$ be an estimative when $e_{3}$ is considered as a controller. We set the error between $e_{3}$ and $e_{3}^{*}$ as

$w_{3}=e_{3}-e_{3}^{*}$.

The time derivative of $V_{2}\left(e_{1}, w_{2}\right)$, leads to:

$$
\begin{aligned}
\dot{V}_{2}\left(e_{1}, w_{2}\right)= & \dot{V}_{1}\left(e_{1}\right)+e_{2}\left(e_{3}-e_{3}^{*}\right)+e_{2} e_{3}^{*}+e_{2}^{2} h_{2}^{2}\left((x, y)_{2}\right)(t) \\
& +e_{2}\left[\eta^{T} \Delta_{2}\left(y_{1}, y_{2}\right)-\theta^{T} \Phi_{2}\left(x_{1}, x_{2}\right)\right] .
\end{aligned}
$$

Let $e_{3}^{*}$ be a virtual adaptive error controller in system (26), such that

$e_{3}^{*}=-e_{2} h_{2}^{2}\left((x, y)_{2}\right)(t)+\hat{\theta}^{T} \Phi_{2}\left(x_{1}, x_{2}\right)-\hat{\eta}^{T} \Delta_{2}\left(y_{1}, y_{2}\right)-c_{2} e_{2}$.

with $c_{2}>0$, being a second constant design parameter. Using (21) and the virtual adaptive error controller given by (27) and (26), leads to the inequality

$$
\begin{aligned}
\dot{V}_{2}\left(e_{1}, w_{2}\right) \leq & e_{1} w_{2}+e_{2} w_{3}+\tilde{\theta}^{T}\left[\Gamma_{\theta}^{-1} \dot{\hat{\theta}}+\Phi_{2}\left(x_{1}, x_{2}\right) e_{2}\right] \\
& +\tilde{\eta}^{T}\left[\Gamma_{\eta}^{-1} \dot{\hat{\eta}}-\Delta_{2}\left(y_{1}, y_{2}\right) e_{2}\right]-c_{2} e_{2}^{2}-c_{1} e_{1}^{2} .
\end{aligned}
$$

We choose the following adaptive law:

$\dot{\hat{\theta}}=-\Gamma_{\theta} \Phi_{2}\left(x_{1}, x_{2}\right) e_{2}$ and

$\dot{\hat{\eta}}=\Gamma_{\eta} \Delta_{2}\left(y_{1}, y_{2}\right) e_{2}$.

Substituting (29) into (28) yields

$\dot{V}_{2}\left(e_{1}, w_{2}\right) \leq \sum_{i=1}^{2} e_{i} w_{i+1}-c_{2} e_{2}^{2}-c_{1} e_{1}^{2}$.

Step $i(2 \leq i \leq n-1)$ : For the $i$ th state of the error dynamics, one can define the error variable $w_{i}$ as:

$w_{i}=e_{i}-e_{i}^{*}$.

Consider $\left(e_{1}, w_{2}, w_{3}, \cdots, w_{i}\right)$ as a subsystem given by

$$
\left\{\begin{array}{l}
\dot{e}_{1}(t)=e_{2}(t)+f_{1}\left(y_{1}, \eta\right)-f_{1}\left(x_{1}, \theta\right) \\
\dot{w}_{2}(t)=e_{3}(t)+f_{2}\left(y_{1}, y_{2}, \eta\right)-f_{2}\left(x_{1}, x_{2}, \theta\right)-\dot{e}_{2}^{*} \\
\dot{w}_{3}(t)=e_{4}(t)+f_{3}\left(y_{1}, y_{2}, y_{3}, \eta\right)-f_{3}\left(x_{1}, x_{2}, x_{3}, \theta\right)-\dot{e}_{3}^{*} \\
\vdots \\
\dot{w}_{i}(t)=e_{i+1}(t)+f_{i}\left(y_{1}, y_{2}, y_{3}, \cdots, y_{i}, \eta\right)-f_{i}\left(x_{1}, x_{2}, x_{3}, \cdots, x_{i}, \theta\right)-\dot{e}_{i}^{*} .
\end{array}\right.
$$

Consider the Lyapunov function defined by

$$
\begin{aligned}
V_{i}\left(e_{1}, w_{2}, \cdots, w_{i}\right)= & \frac{1}{2} e_{i}^{2}+V_{i-1}\left(e_{1}, w_{2}, \cdots, w_{i-1}\right) \\
& +\int_{t_{0}}^{t} e_{i}^{2}(\tau) h_{i}^{2}\left((x, y)_{i}\right)(\tau) d \tau .
\end{aligned}
$$

where $(x(\tau), y(\tau))_{i}=\left(x_{1}(\tau), \cdots, x_{i}(\tau), y_{1}(\tau), \cdots, y_{i}(\tau)\right)$. Let $e_{i+1}^{*}$ be an estimative when $e_{i}$ is considered as a controller. We set the error between $e_{i+1}$ and $e_{i+1}^{*}$ as

$w_{i+1}=e_{i+1}-e_{i+1}^{*}$.

The time derivative of $V_{i}\left(e_{1}, w_{2}, \cdots, w_{i}\right)$, leads to:

$$
\begin{aligned}
\dot{V}_{i}\left(e_{1}, w_{2}, \cdots, w_{i}\right)= & \dot{V}_{i-1}\left(e_{1}, w_{2}, \cdots, w_{i-1}\right) \\
& +e_{i}\left(e_{i+1}-e_{i+1}^{*}\right)+e_{i} e_{i+1}^{*}+e_{i}^{2} h_{i}^{2}\left((x, y)_{i}\right)(t) \\
& +e_{i}\left[\eta^{T} \Delta_{i}\left(y_{1}, \cdots, y_{i}\right)-\theta^{T} \Phi_{i}\left(x_{1}, \cdots, x_{i}\right)\right] .
\end{aligned}
$$

Let $e_{i+1}^{*}$ be a virtual adaptive error controller in system (35), such that

$$
e_{i+1}^{*}=-e_{i} h_{i}^{2}\left((x, y)_{i}\right)(t)+\hat{\theta}^{T} \Phi_{i}\left(x_{1}, \cdots, x_{i}\right)-\hat{\eta}^{T} \Delta_{i}\left(y_{1}, \cdots, y_{i}\right)-c_{i} e_{i} \text {, }
$$

with $c_{i}>0$, being an $i$ th constant design parameter.

Using the recursive precedent inequelity on $\dot{V}_{i-1}\left(e_{1}, w_{2}, \cdots, w_{i-1}\right)$ and the virtual adaptive error controller given by (36) in (35), leads to the inequality

$$
\dot{V}_{i}\left(e_{1}, w_{2}, \cdots, w_{i}\right) \leq \sum_{j=1}^{i} e_{j} w_{j+1}+\tilde{\theta}^{T}\left[\Gamma_{\theta}^{-1} \dot{\hat{\theta}}+\Phi_{i}\left(x_{1}, \cdots, x_{i}\right) e_{i}\right]
$$




$$
+\tilde{\eta}^{T}\left[\Gamma_{\eta}^{-1} \dot{\hat{\eta}}-\Delta_{i}\left(y_{1}, \cdots, y_{i}\right) e_{i}\right]-\sum_{j=1}^{i} c_{j} e_{j}^{2} .
$$

We choose the following adaptive law defined by

$\dot{\hat{\theta}}=-\Gamma_{\theta} \Phi_{i}\left(x_{1}, \cdots, x_{i}\right) e_{i}$ and

$\dot{\hat{\eta}}=\Gamma_{\eta} \Delta_{i}\left(y_{1}, \cdots, y_{i}\right) e_{i}$.

When substituting (38) into (37), it yields

$\dot{V}_{i}\left(e_{1}, w_{2}, \cdots, w_{i}\right) \leq \sum_{j=1}^{i} e_{j} w_{j+1}-\sum_{j=1}^{i} c_{j} e_{j}^{2}$.

Step $n$ : The final design step is to set the state feedback input wich make the system (3) asymptotically stable in finite-time. Then, we define the error variable $w_{n}$, as

$w_{n}=e_{n}-e_{n}^{*}$.

Consider the $\left(e_{1}, w_{2}, w_{3}, \cdots, w_{n}\right)$ subsystem given by:

$$
\left\{\begin{array}{l}
\dot{e}_{1}(t)=e_{2}(t)+f_{1}\left(y_{1}, \eta\right)-f_{1}\left(x_{1}, \theta\right) \\
\dot{w}_{2}(t)=e_{3}(t)+f_{2}\left(y_{1}, y_{2}, \eta\right)-f_{2}\left(x_{1}, x_{2}, \theta\right)-\dot{e}_{2}^{*} \\
\dot{w}_{3}(t)=e_{4}(t)+f_{3}\left(y_{1}, y_{2}, y_{3}, \eta\right)-f_{3}\left(x_{1}, x_{2}, x_{3}, \theta\right)-\dot{e}_{3}^{*} \\
\vdots \\
\dot{w}_{n}(t)=f_{n}\left(y_{1}, y_{2}, y_{3}, \cdots, y_{n}, \eta\right)-f_{n}\left(x_{1}, x_{2}, x_{3}, \cdots, x_{n}, \theta\right)+u-\dot{e}_{n}^{*} .
\end{array}\right.
$$

Then, we shall consider the Lyapunov function defined by

$$
\begin{aligned}
V_{n}\left(e_{1}, w_{2}, \cdots, w_{n}\right)= & \frac{1}{2} e_{n}^{2}+V_{n-1}\left(e_{1}, w_{2}, \cdots, w_{n-1}\right) \\
& +\int_{t_{0}}^{t} e_{n}^{2}(\tau) h_{n}^{2}\left((x, y)_{n}\right)(\tau) d \tau .
\end{aligned}
$$

Therefore, the time derivative of $V_{i}\left(e_{1}, w_{2}, \cdots, w_{n}\right)$, leads to:

$$
\begin{aligned}
\dot{V}_{n}\left(e_{1}, w_{2}, \cdots, w_{n}\right)= & \dot{V}_{n-1}\left(e_{1}, w_{2}, \cdots, w_{n-1}\right)+e_{n} u+e_{n}^{2} h_{n}^{2}\left((x, y)_{n}\right)(t), \\
& +e_{n}\left[\eta^{T} \Delta_{n}\left(y_{1}, \cdots, y_{n}\right)-\theta^{T} \Phi_{n}\left(x_{1}, \cdots, x_{n}\right)\right],
\end{aligned}
$$

where $\dot{V}_{n}$ is a negative definite function on $\mathbb{R}^{n}$.

Thus, by Lyapunov stability theory [12], the dynamic errors (3) is asymptotically stable for all initial conditions $e_{i}\left(t_{0}\right)$ and in particular $e_{i}(0), \forall i \in \mathbb{N}$. The virtual control is given by the following form

$u=-e_{n} h_{n}^{2}\left((x, y)_{n}\right)(t)+\hat{\theta}^{T} \Phi_{n}\left(x_{1}, \cdots, x_{n}\right)-\hat{\eta}^{T} \Delta_{i}\left(y_{1}, \cdots, y_{n}\right)-c_{n} e_{n}$,

with $c_{n}>0$, being an $n$th constant design parameter. Which leads to the final inequality

$$
\dot{V}_{n}\left(e_{1}, w_{2}, \cdots, w_{n}\right) \leq \sum_{j=1}^{n-1} e_{j} w_{j+1}-\sum_{j=1}^{n} c_{j} e_{j}^{2} .
$$

Then $\dot{V}_{n}$ is a negative definite function on $\mathbb{R}^{n}$, with the well chosen following adaptive parameters

$$
\begin{aligned}
& \dot{\hat{\theta}}=-\Gamma_{\theta} \Phi_{n}\left(x_{1}, \cdots, x_{n}\right) e_{n} \text { and } \\
& \dot{\hat{\eta}}=\Gamma_{\eta} \Delta_{n}\left(y_{1}, \cdots, y_{n}\right) e_{n},
\end{aligned}
$$

and we obtain the finite-time chaos synchronization between both systems (1) and (2) by the asymptotic stability of (41) in finite-time.

\section{Simulation Study}

In this section, we will give two examples to illustrate efficiency of our control algorithm approach proposed in this paper. Many other effective methods and techniques have been developed on the same examples, such as feedback approach [41], sliding-mode [37] and backstepping design technique $[26,36]$.

\subsection{Example 1: Two Genesio-Tesi System}

In this example, the control algorithm will be used to synchronize two Genesio-Tesi systems with different unknown parameters and different initial conditions. The mathematical expressions are illustrated by the following nonlinear equations $[8,17,40]$.

The master system is described by the Genesio-Tesi system dynamics which correspond 


$$
\left\{\begin{array}{l}
\dot{x}_{1}(t)=x_{2} \\
\dot{x}_{2}(t)=x_{3} \\
\dot{x}_{3}(t)=\theta_{3} x_{3}+\theta_{2} x_{2}+\theta_{1} x_{1}+x_{1}^{2}
\end{array}\right.
$$

where $x_{1}, x_{2}, x_{3}$ are state variables and $\theta_{1}, \theta_{2}, \theta_{3}$ are negative, constant parameters of the system. The slave system is taken as follows:

$$
\left\{\begin{array}{l}
\dot{y}_{1}(t)=y_{2} \\
\dot{y}_{2}(t)=y_{3} \\
\dot{y}_{3}(t)=\eta_{3} y_{3}+\eta_{2} y_{2}+\eta_{1} y_{1}+y_{1}^{2}+u,
\end{array}\right.
$$

where $y_{1}, y_{2}, y_{3}$ are state variables, $\eta_{1}, \eta_{2}, \eta_{3}$ are other negative, constant parameters and $u$ is the controller to be designed.

After some calculations, the following dynamic errors of the system $(e=y-x)$ is obtained such as

$$
\left\{\begin{array}{l}
\dot{e}_{1}(t)=e_{2} \\
\dot{e}_{2}(t)=e_{3} \\
\dot{e}_{3}(t)=\eta_{3} y_{3}-\theta_{3} x_{3}+\eta_{2} y_{2}-\theta_{2} x_{2}+\eta_{1} y_{1}-\theta_{1} x_{1}+y_{1}^{2}-x_{1}^{2}+u
\end{array}\right.
$$

First, we consider the stability of the system without any parameters

$\dot{e}_{1}(t)=e_{2}$.

We choose the Lyapunov function defined by $V_{1}\left(e_{1}\right)=\frac{1}{2} e_{1}^{2}$, which its time derivative is given by

$\dot{V}_{1}\left(e_{1}\right)=e_{1} e_{2} \leq e_{1} w_{2}+e_{1} e_{2}^{*}$,

where $w_{2}=e_{2}-e_{2}^{*}$ and $e_{2}^{*}$ is the control error defined by

$e_{2}^{*}=-c_{1} e_{1}$.

In this situation, $c_{1}>0$ is an adequat chosen design parameter. This leads to

$\dot{\mathrm{V}}_{1}\left(\mathrm{e}_{1}\right) \leq \mathrm{e}_{1} \mathrm{~W}_{2}-\mathrm{c}_{1} \mathrm{e}_{1}^{2}$.

We take the subsystem $\left(e_{1}, w_{2}\right)$, without parameters, defined by:

$\left\{\begin{array}{l}\dot{\mathrm{e}}_{1}(\mathrm{t})=\mathrm{e}_{2}(\mathrm{t}) \\ \dot{\mathrm{w}}_{2}(\mathrm{t})=\mathrm{e}_{3}(\mathrm{t})-\dot{\mathrm{e}}_{2}^{*},\end{array}\right.$

and we define the Lyapynov function associated to (54) as

$\mathrm{V}_{2}\left(\mathrm{e}_{1}, \mathrm{w}_{2}\right)=\frac{1}{2} \mathrm{e}_{2}^{2}+\mathrm{V}_{1}\left(\mathrm{e}_{1}\right)$.

We set $w_{3}=e_{3}-e_{3}^{*}$ and $e_{3}^{*}$ is the control error such that $e_{3}^{*}=-c_{2} e_{2}$, where $c_{2}>0$ is a second chosen parameter. Thus, the time derivative of (55), using (53) is as the following:

$\dot{\mathrm{V}}_{2}\left(\mathrm{e}_{1}, \mathrm{w}_{2}\right) \leq \mathrm{e}_{1} \mathrm{w}_{2}+\mathrm{e}_{2} \mathrm{w}_{3}-\mathrm{c}_{1} \mathrm{e}_{1}^{2}-\mathrm{c}_{2} \mathrm{e}_{2}^{2}$.

We take the subsystem $\left(e_{1}, w_{2}, w_{3}\right)$, with unknown parameters, given by:

$\left\{\begin{array}{l}\dot{e}_{1}(t)=e_{2}(t) \\ \dot{w}_{2}(t)=e_{3}(t)-\dot{e}_{2}^{*} \\ \dot{w}_{3}(t)=u+\eta_{3} y_{3}-\theta_{3} x_{3}+\eta_{2} y_{2}-\theta_{2} x_{2}+\eta_{1} y_{1}-\theta_{1} x_{1}+y_{1}^{2}-x_{1}^{2}-\dot{e}_{3}^{*} .\end{array}\right.$

We construct the Lyapunov function defined by

$$
\begin{aligned}
V_{3}\left(e_{1}, w_{2}, w_{3}\right)= & \frac{1}{2} e_{3}^{2}+V_{2}\left(e_{1}, w_{2}\right) \\
& +\int_{t_{0}}^{t} e_{3}^{2}(\tau) h_{3}^{2}\left((x, y)_{3}\right)(\tau) d \tau \\
& +\frac{1}{2} \tilde{\theta}^{\mathrm{T}} \Gamma_{\theta}^{-1} \tilde{\theta}+\frac{1}{2} \tilde{\eta}^{\mathrm{T}} \Gamma_{\eta}^{-1} \tilde{\eta},
\end{aligned}
$$



$\hat{\eta}$.

where $\theta=\left(\theta_{1}, \theta_{2}, \theta_{3}\right)^{T} \in \mathbb{R}^{3 \times 1}, \eta=\left(\eta_{1}, \eta_{2}, \eta_{3}\right)^{T} \in \mathbb{R}^{3 \times 1}$ and $\tilde{\theta}=\theta-\hat{\theta}, \tilde{\eta}=\eta-$

The time derivative of $V_{3}\left(e_{1}, w_{2}, w_{3}\right)$, leads to:

$$
\begin{aligned}
\begin{array}{l}
\dot{V}_{3}\left(e_{1}, w_{2}, w_{3}\right)=e_{1} e_{2}+e_{2} e_{3}+e_{3} u+e_{3}^{2} h_{3}^{2}\left((x, y)_{3}\right)(t) \\
\eta_{1} \mathrm{y}_{1}-\theta_{1} \mathrm{x}_{1}+\mathrm{y}_{1}^{2}-\mathrm{x}_{1}^{2}
\end{array} & +\tilde{\theta}^{T} \Gamma_{\theta}^{-1} \dot{\hat{\theta}}+\tilde{\eta}^{T} \Gamma_{\eta}^{-1} \hat{\hat{\eta}}+\mathrm{e}_{3}\left[\eta_{3} \mathrm{y}_{3}-\theta_{3} \mathrm{x}_{3}+\eta_{2} \mathrm{y}_{2}-\theta_{2} \mathrm{x}_{2}+\right.
\end{aligned}
$$

We consider the chosen scaling function $(x, y)_{3} \mapsto h_{3}^{2}\left((x, y)_{3}\right)$ with $(x, y)_{3}=$ $\left(x_{1}, x_{2}, x_{3}, y_{1}, y_{2}, y_{3}\right)$, such that

$\mathbb{R}^{3} \times \mathbb{R}^{3} \rightarrow \mathbb{R}^{+}:(\mathrm{x}, \mathrm{y})_{3} \mapsto \mathrm{h}_{3}^{2}\left((\mathrm{x}, \mathrm{y})_{3}\right)=\mathrm{x}_{1}^{3}-\mathrm{y}_{1}^{3}$.

Equation (60) is a smoooth function verifying the Assumption (9) and we also have

$$
\lim _{(0,0)} h_{3}\left((x, y)_{3}\right)=0
$$

Then, we design the following virtual adaptive recursive controller given by

$$
\begin{aligned}
u= & \hat{\theta}_{3} x_{3}+\hat{\theta}_{2} x_{2}+\hat{\theta}_{1} x_{1} \\
& -\hat{\eta}_{3} y_{3}-\hat{\eta}_{2} y_{2}-\hat{\eta}_{1} y_{1}+x_{1}^{2} \\
& -\mathrm{y}_{1}^{2}-\mathrm{c}_{3} \mathrm{e}_{3}-\mathrm{e}_{3} \mathrm{~h}_{3}^{2}\left((\mathrm{x}, \mathrm{y})_{3}\right)(\mathrm{t}),
\end{aligned}
$$

with $c_{3}>0$, being a constant design parameter, with

$\Phi_{3}\left(x_{1}, x_{2}, x_{3}\right)=\left(x_{1}, x_{2}, x_{3}\right)^{T}$ and

$\Delta_{3}\left(y_{1}, y_{2}, y_{3}\right)=\left(y_{1}, y_{2}, y_{3}\right)^{T}$,

and the adaptative laws, choosing respectively the adequate rates of adaptation as

$\Gamma_{\theta}=\left(\begin{array}{lll}k_{1} & 0 & 0 \\ 0 & k_{2} & 0 \\ 0 & 0 & k_{3}\end{array}\right) \quad$ and $\quad \Gamma_{\eta}=\left(\begin{array}{lll}k_{1}^{\prime} & 0 & 0 \\ 0 & k_{2}^{\prime} & 0 \\ 0 & 0 & k_{3}^{\prime}\end{array}\right)$

with

$k_{1}=2, k_{2}=1, k_{3}=-1, k_{1}^{\prime}=-1.5, k_{2}^{\prime}=1, k_{3}^{\prime}=1$,

and

$\dot{\hat{\theta}}=\left(\begin{array}{l}\dot{\hat{\theta}}_{1} \\ \dot{\hat{\theta}}_{2} \\ \hat{\hat{\theta}}_{3}\end{array}\right)=-\Gamma_{\theta} \Phi_{3}\left(x_{1}, x_{2}, x_{3}\right) e_{3}=\left(\begin{array}{l}-k_{1} x_{1} e_{3} \\ -k_{2} x_{2} e_{3} \\ -k_{3} x_{3} e_{3}\end{array}\right)$

and

$$
\dot{\hat{\eta}}=\left(\begin{array}{l}
\dot{\hat{\eta}}_{1} \\
\dot{\hat{\eta}}_{2} \\
\dot{\hat{\eta}}_{3}
\end{array}\right)=\Gamma_{\eta} \Delta_{3}\left(y_{1}, y_{2}, y_{3}\right) e_{3}=\left(\begin{array}{l}
k_{1}^{\prime} y_{1} e_{3} \\
k_{2}^{\prime} y_{2} e_{3} \\
k_{3}^{\prime} y_{3} e_{3}
\end{array}\right)
$$

Substituting (60),(61),(62), (63), (65) and (66) into (59) and using inequality (56), leads to

$\dot{V}_{3}\left(e_{1}, w_{2}, w_{3}\right) \leq e_{1} w_{2}+e_{2} w_{3}-c_{1} e_{1}^{2}-c_{2} e_{2}^{2}-c_{3} e_{3}^{2}$.

Then, $\dot{V}_{n}$ is a negative definite function on $\mathbb{R}^{n}$, for any initial conditions and with different unknown parameters and the subsystem $\left(e_{1}, w_{2}, w_{3}\right)$ is asymptotically stable. This leads us to the finite-time stabilization of the dynamic errors (49) and eventually to the finite-time synchronization of both chaotic systems (47) and (48).

For the numerical simulations, the Euler method is used to verify the efficiency of the proposed method on two Genesio-Tesi systems with different unknown parameters and unequal initial conditions. Thus, the $3 D$ systems (47) and (48) are chaotic for $\theta_{1}=$ $-6, \theta_{2}=-2.92, \theta_{3}=-1.2$ and $\eta_{1}=-5, \eta_{2}=-3, \eta_{3}=-1$, with the initial values of the master system $x_{1}(0)=0, x_{2}(0)=1, x_{3}(0)=1$ and the initial values of the slave system $y_{1}(0)=-1, y_{2}(0)=0, y_{3}(0)=0.5$ with $c_{3}=500$ and the initial values of the estimated parameters $\hat{\theta}_{1}(0)=-6, \hat{\theta}_{3}(0)=-2.92, \hat{\theta}_{3}(0)=-1.2, \hat{\eta}_{1}(0)=-5, \hat{\eta}_{2}(0)=$ $-3, \hat{\eta}_{3}(0)=-1$. 
Figure 1 and Figure 2 display the chaotic behavior of respectively the master and slave system. Figure 3 dispalys the synchronization errors of systems (47) and (48) and the convergence of the synchronization error. Figure 4 dispalys the time response of states for the master system (47) and the states for the slave system (48). Figure 5 shows that the estimated values $\left(\hat{\theta}_{1}, \hat{\theta}_{3}, \hat{\theta}_{3}, \hat{\eta}_{1}, \hat{\eta}_{2}, \hat{\eta}_{3}\right)$ of unknown parameters of the systems (47) and (48) converge in finite-time to $\left(\theta_{1}=-6, \theta_{2}=-1, \theta_{3}=-1.2, \eta_{1}=-5, \eta_{2}=-3, \eta_{3}=\right.$ -1 ), respectively as $t \rightarrow+\infty$. It can be seen from the figures a rapid convergence to zero synchronization errors, verifying the effectiveness of the method we propose.

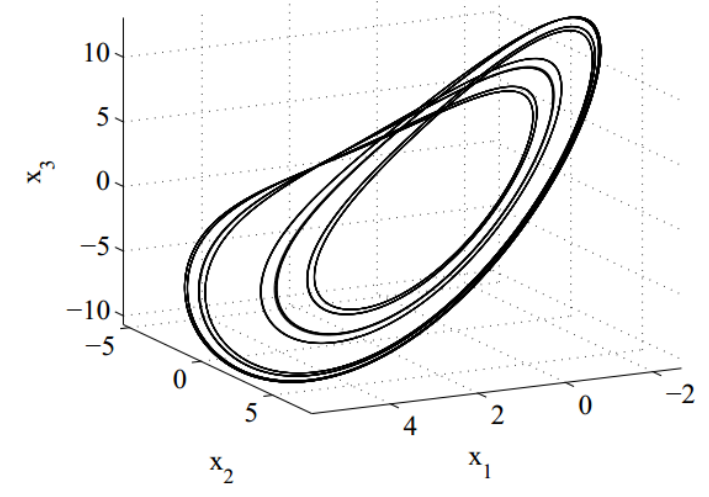

Figure 1. Chaotic Behavior of Genesio-Tesi Master System with $\theta=$ $(-6,-2.92,-1.2), x(0)=(0,1,1)$

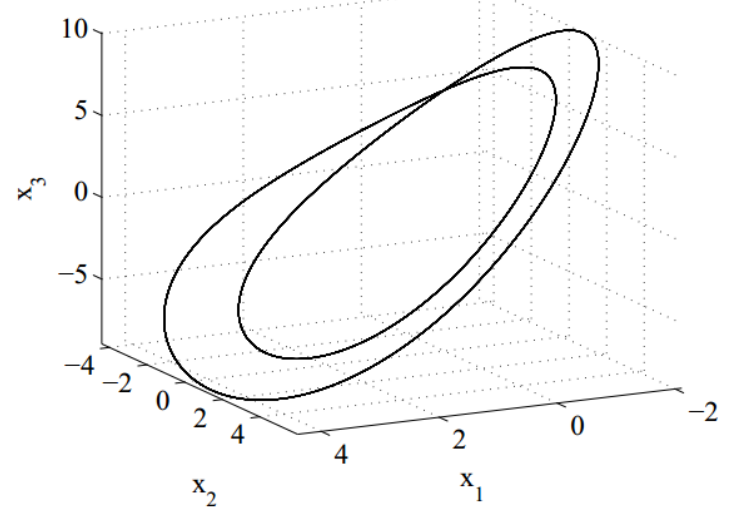

Figure 2. Chaotic Behavior of Genesio-Tesi Slave System with $\eta=$ $(-5,-3,-1), y(0)=(-1,0,0.5)$ 


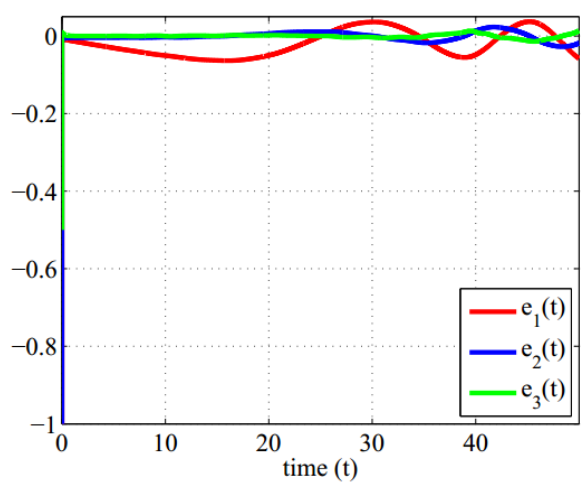

Figure 3. Time History of the Synchronization Error States $\mathbf{e}_{1}, \mathbf{e}_{2}, \mathbf{e}_{3}$ between Two Chaotic Systems Genesio-Tesi, with Different Unknown Parameters)
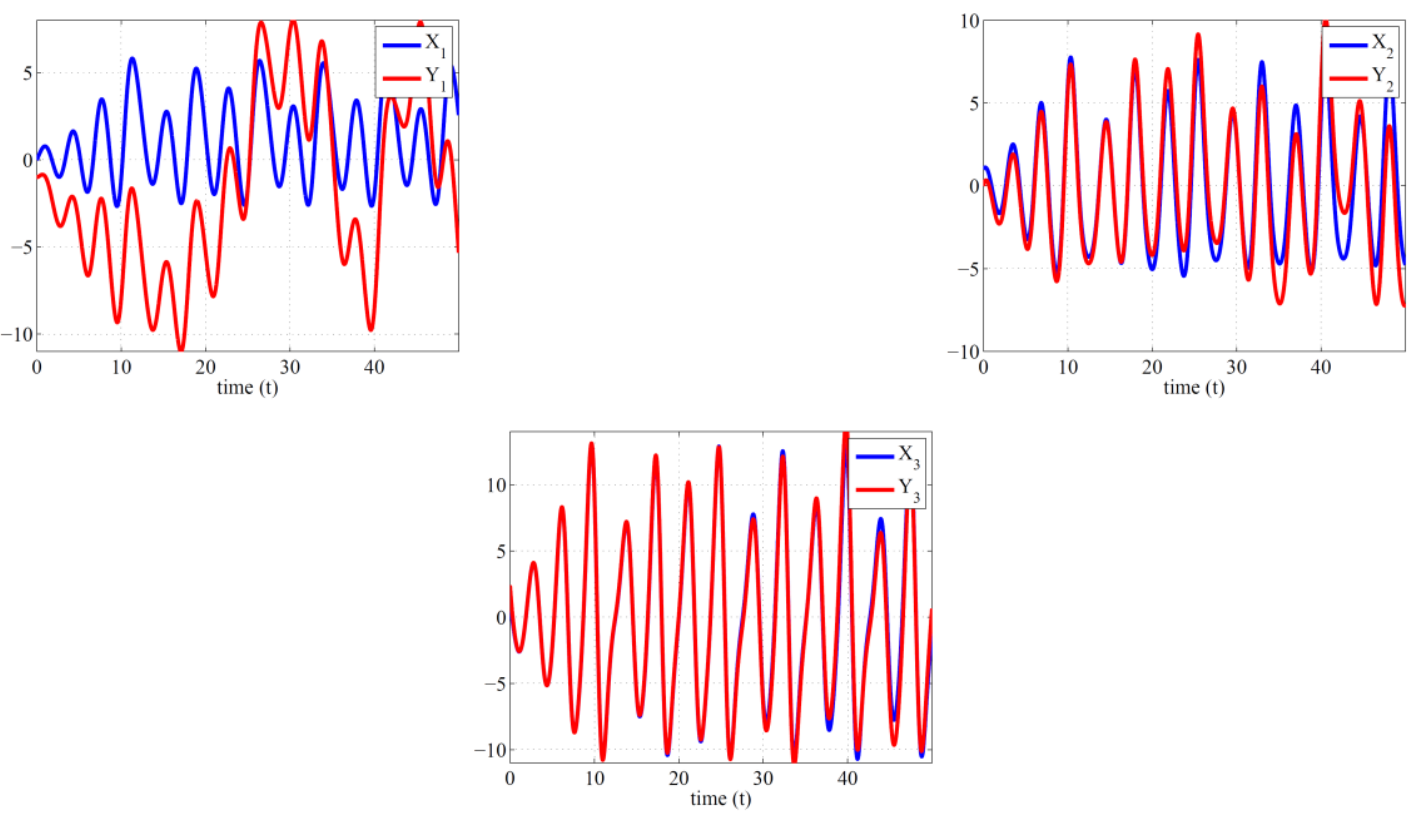

Figure 4. Time Evolutions of the States of the Master System (47) and the Slave System (48) With the Controller (61) (Synchronization of two Chaotic Systems Genesio-Tesi With Different Unknown Parameters)

\subsection{Example 2: Genesio-Tesi and Coullet systems}

In this example, it is assumed that Genesio-Tesi is the master system and Coullet is the slave system $[13,30,35]$. The master system is described previously in (47) from example 1 and thus, we introduce the following slave dynamics:

$$
\left\{\begin{array}{l}
\dot{y}_{1}(t)=y_{2} \\
\dot{y}_{2}(t)=y_{3} \\
\dot{y}_{3}(t)=\eta_{3} y_{3}+\eta_{2} y_{2}+\eta_{1} y_{1}-y_{1}^{3}+u .
\end{array}\right.
$$

The dynamic errors is obtained as following

$$
\left\{\begin{array}{l}
\dot{e}_{1}(t)=e_{2} \\
\dot{e}_{2}(t)=e_{3} \\
\dot{e}_{3}(t)=\eta_{3} y_{3}-\theta_{3} x_{3}+\eta_{2} y_{2}-\theta_{2} x_{2}+\eta_{1} y_{1}-\theta_{1} x_{1}-y_{1}^{3}-x_{1}^{2}+u
\end{array}\right.
$$



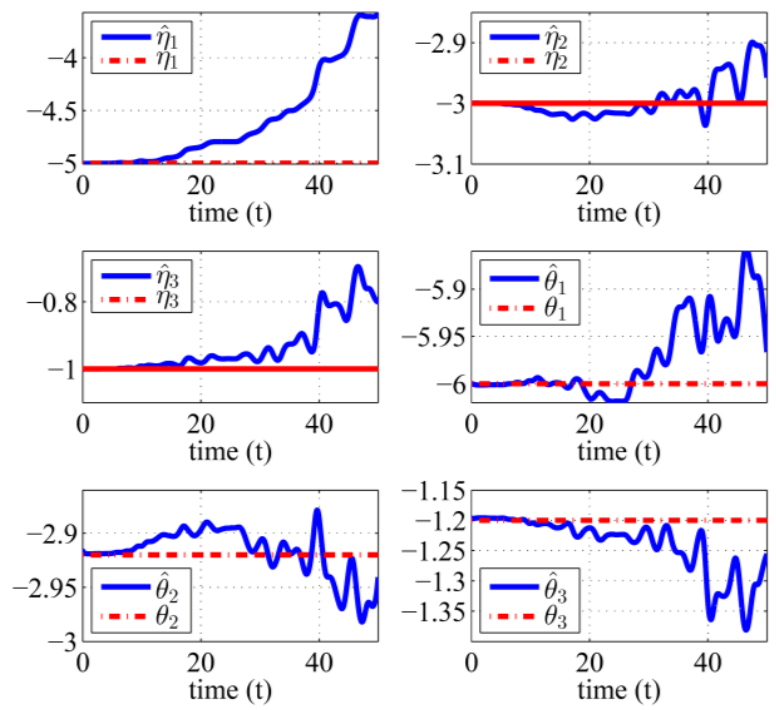

Figure 5. Parameter Estimation Results (Synchronization in Finite-Time of the Two Chaotic Systems Genesio-Tesi, With Different Unknown Parameters)

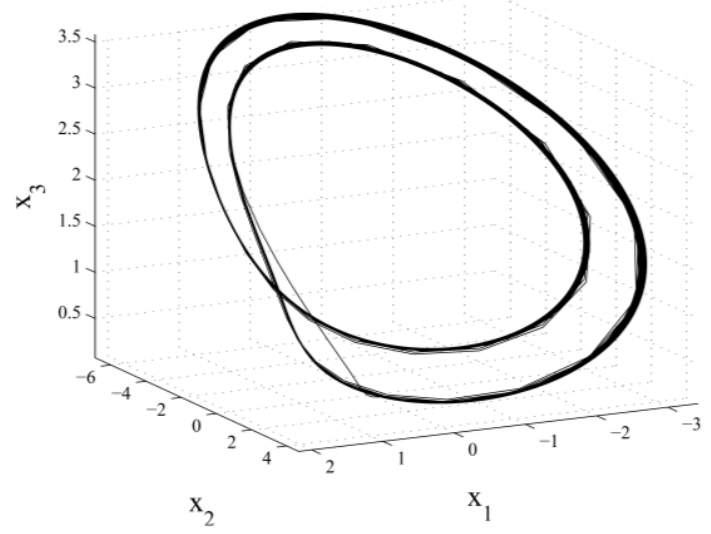

Figure 6. Chaotic Behavior of Coullet System with $\eta=(7,3.5,2.3), y(0)=$ $(0.1,-0.1,0.1)$

For the controller design algorithm, we keep the same step 1 and step 2, of the example 1 . Then we consider the subsystem $\left(e_{1}, w_{2}, w_{3}\right)$, with unknown different parameters, given by

$$
\left\{\begin{array}{l}
\dot{e}_{1}(t)=e_{2}(t) \\
\dot{w}_{2}(t)=e_{3}(t)-\dot{e}_{2}^{*} \\
\dot{w}_{3}(t)=u+\eta_{3} y_{3}
\end{array}\right.
$$

We consider the Lyapunov function given in (58). The time derivative of (58) leads to

$$
\begin{aligned}
\dot{V}_{3}\left(e_{1}, w_{2}, w_{3}\right)= & e_{1} e_{2}+e_{2} e_{3}+e_{3} u+e_{3}^{2} h_{3}^{2}\left((x, y)_{3}\right)(t)+\tilde{\theta}^{T} \Gamma_{\theta}^{-1} \dot{\hat{\theta}} \\
& +\tilde{\eta}^{T} \Gamma_{\eta}^{-1} \dot{\hat{\eta}} \\
& +e_{3}\left[\eta_{3} y_{3}-\theta_{3} x_{3}+\eta_{2} y_{2}-\theta_{2} x_{2}+\eta_{1} y_{1}-\theta_{1} x_{1}-y_{1}^{3}-x_{1}^{2}\right] .
\end{aligned}
$$

Consider the scaling smooth function $(x, y)_{3} \mapsto h_{3}^{2}\left((x, y)_{3}\right)$ with $(x, y)_{3}=$ $\left(x_{1}, x_{2}, x_{3}, y_{1}, y_{2}, y_{3}\right)$, such that

$$
\mathbb{R}^{3} \times \mathbb{R}^{3} \rightarrow \mathbb{R}^{+}:(x, y)_{3} \mapsto h_{3}^{2}\left((x, y)_{3}\right)=x_{1}^{2}-y_{1}^{2}
$$

The designed controller is also given by (61), choosing the adaptive laws (62), (63), (65) and (66) with 
$k_{1}=5, k_{2}=1, k_{3}=1, k_{1}^{\prime}=-0.5, k_{2}^{\prime}=1, k_{3}^{\prime}=1$.

The time derivative of (72), leads to the same inéquality seen in (67).

In the next simulation, we discuss the result for the synchronization between both Genesio-Tesi and Coullet systems which are topologically different systems. Also, they are known to be difficult to synchronize in finite-time. The $3-D$ systems from (47) and (68) are chaotic for $\theta_{1}=-6, \theta_{2}=-2.92, \theta_{3}=-1.2$ and $\eta_{1}=7, \eta_{2}=3.5, \eta_{3}=2.5$, with the initial values of the master system $x_{1}(0)=0, x_{2}(0)=1, x_{3}(0)=1$, the initial values of the slave system $y_{1}(0)=0.1, y_{2}(0)=-0.1, y_{3}(0)=0.1$ with $c_{3}=250$ and the initial values of the estimated parameters $\hat{\theta}_{1}(0)=-6, \hat{\theta}_{3}(0)=-2.92, \hat{\theta}_{3}(0)=$ $-1.2, \hat{\eta}_{1}(0)=7, \hat{\eta}_{2}(0)=3.5, \hat{\eta}_{3}(0)=2.5$.

Figure 6 displays the Coullet chaotic behavior. In this figure, we notice the importance of the initial conditions in the implementation. Then, Figure 7 displays the synchronization errors of both systems (47) and (68). Figure 8 dispalys the time response of states for the master system (47) and the states for the slave system (68). Figure 9 shows that the estimated values $\left(\hat{\theta}_{1}, \hat{\theta}_{3}, \hat{\theta}_{3}, \hat{\eta}_{1}, \hat{\eta}_{2}, \hat{\eta}_{3}\right)$ of unknown parameters of the systems (47) and (48) converge in finite-time to $\left(\theta_{1}=-6, \theta_{2}=-1, \theta_{3}=-1.2, \eta_{1}=7, \eta_{2}=3.5, \eta_{3}=\right.$ $2.5)$, respectively as $t \rightarrow+\infty$.

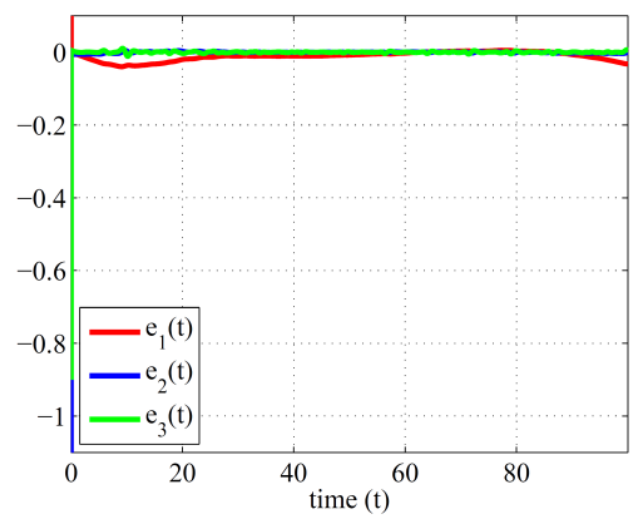

Figure 7. Time History of the Synchronization Error States $\mathbf{e}_{1}, \mathbf{e}_{2}, \mathbf{e}_{3}$ between Two Chaotic Systems Genesio-Tesi and Coullet with Different Unknown Parameters 

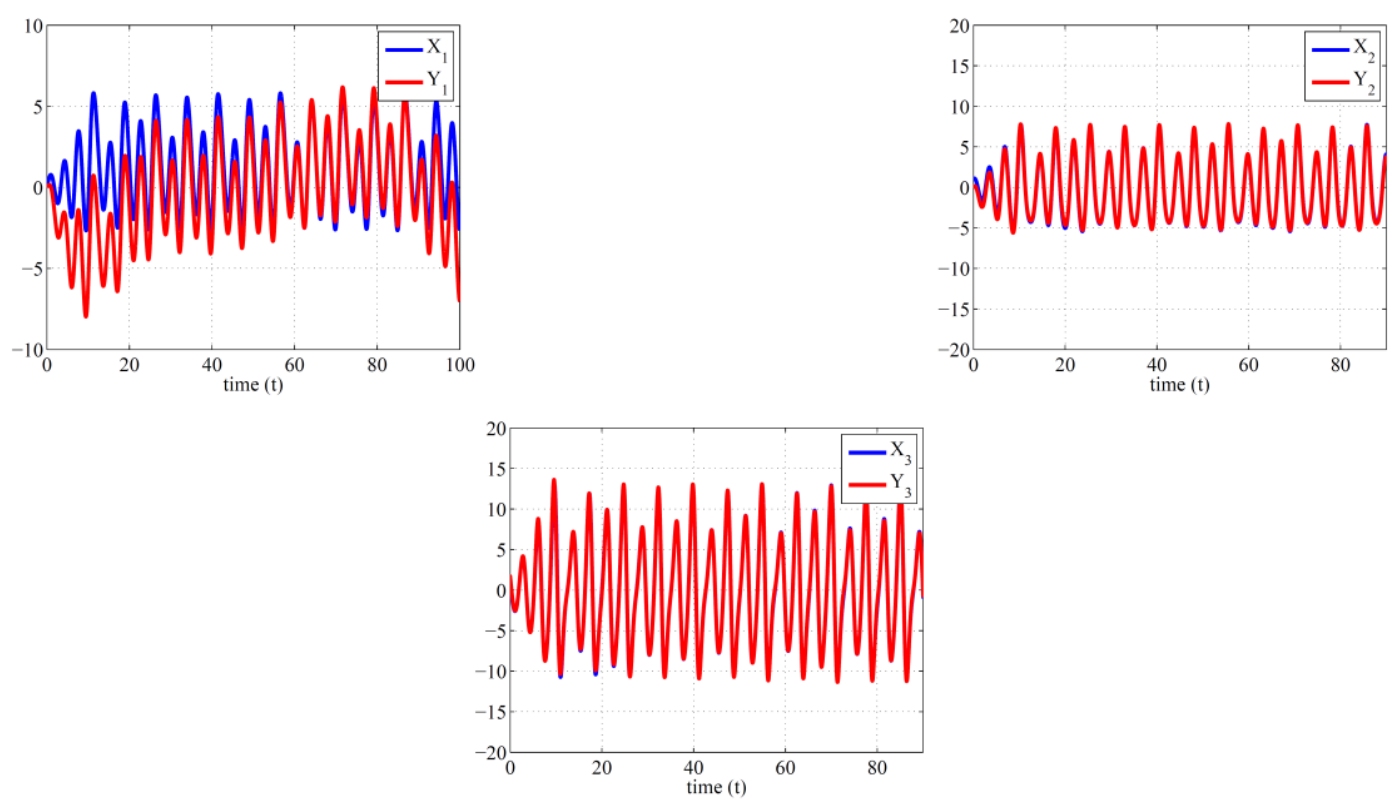

Figure 8. Time Evolutions of the States of the Master System (47) and the Slave System (68) With the Controller (61) (Synchronization in Finite-Time of Two Chaotic Systems Genesio-Tesi and Coullet, With Different Unknown Parameters)

The simulation results indicate that the proposed constructive method for a virtual synchronizing controller in finite-time gives interesting results even if the systems are topologically different.

\subsection{Discussion}

During simulation using MatLAB software, there still exist a sensitive dependence in the initial conditions of the states and parameters initialization for both systems given in examples 1 and 2, which made us to initialize the parameters to be estimated to their real values. As a conclusion, this encourages us to consider, in further works, some ameliorations of our proposed algorithm in case where the parameters are subject to disturbances. 

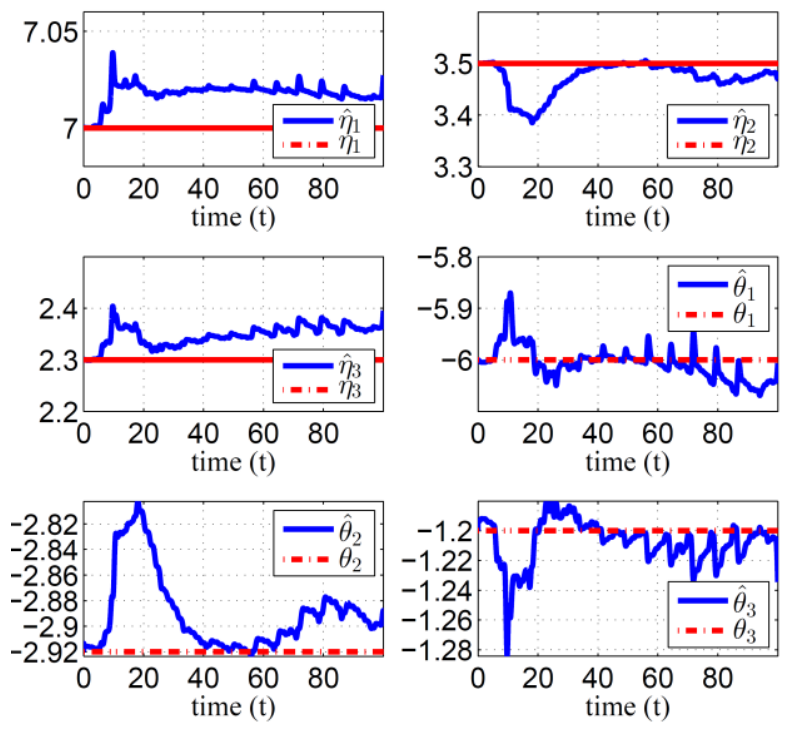

\section{Figure 9. Parameter Estimation Results, Synchronization In Finite-Time Of The Two Chaotic Systems Genesio-Tesi and Coullet, With Different Unknown Parameters}

\section{Conclusion}

In this research work, we have investigated and proposed an algorithm able to synchronize, in finite-time, two chaotic systems in a master-slave configuration. This algorithm was evaluated using 3D identical and non-identical chaotic systems using Genesio-Tesi and Coullet topology. We suggest using an adaptive control method when system parameters are unknown or uncertain. The simulation results presented for both examples validate the worth of the proposed synchronization algorithm since they could illustrate effectiveness of our method.

For the sake of simplicity, we have restricted our consideration for unknown parameters case. Our method, however, can straightforwardly be extended to the cases in which these parameters are uncertain and could be perturbed. Then, a study on the system sensitivity could be completed in future work considering initial conditions and perturbation. It could give more insight for increasing robustness and performance of the approach.

Based on the Lyapunov finite-time stability theory, the synchronization will be achieved even in presence of unknown parameters by choosing a proper adaptation law and by designing a virtual adaptive controller, using a recursive approach. Consequently, the extension of the control strategy to higher-dimensional chaotic systems and to systems with uncertain parameters is promising.

\section{References}

[1] O. Morgul and M. Feki, "Synchronization of chaotic systems by using occasional coupling", Physics, Revue, vol. E55, pp. 5004-5010, (1997).

[2] S. P. Bhat and D. S. Bernstein, "Finite-time stability of homogeneous systems", In Proceedings of the American Control Conference, Albuquerque, NM, 4-6 Jun 1997, (1997), pp. 2513-2514.

[3] S P Bhat and D. S. Bernstein, "Finite-time stability of continuous autonomous systems", SIAM Jouranal on Control and Optimization, vol. 38, (2000), pp. 751-766.

[4] S. Bhalekar and V. Daftardar-Gejji, "Synchronization of different fractional order chaotic systems using active control", Article in Press, (2010).

[5] J. Cai and M. Lin, "Finite-time synchronization of non-autonomous chaotic systems with unknown parameters", Chaos-Fractals Theories and Applications (IWCFTA), Kunming, Yunnan, (2010), pp.8-13. 
[6] T. L. Carroll and L. M. Pecora, "Synchronization chaotic circuits", IEEE Trans. Circuits Syst., vol. 38, no. 4, (1991), pp. 453-456.

[7] G. Chen and X. Dong, "From Chaos to Order - Methodologies, Perspectives and Applications", World Scientific, Singapore, (1998).

[8] P. Coullet, C. Tresser and A. Arneodo, "Transition to stochasticity for a class of forced oscillators", Phys. Lett. A., vol. 72, (1979), pp. 268-270.

[9] H. Fujisaka and T. Yamada, "Stability theory of synchronized motion in coupled-oscillator systems", Progress of Theoretical Physics, vol. 63, (1983), pp. 32-47.

[10] S. K. Han, C. Kerrer and Y. Kuramoto, "D-phasing and bursting in coupled neural oscillators", Phys. Rev. Lett., vol. 75, (1995), pp. 3190-3193.

[11] Z. Guangb, Z. S. C Lan and H. S. Li, "Complexity of an SIR epidemic dynamics model with impulsive vaccination control”, Chaos, Solutions and Fractals, vol. 26, (2005), pp.495-505.

[12] W. Hahn, "The Stability of Motion", Berlin, Germany, Springer-Verlag (1967).

[13] J. B. Hu, Y. Han and L. D Zhao, "Synchronization in the Genesio-Tesi and Coullet systems using the Backstepping Approach", International Symposium on Nonlinear Dynamics, Journal of Physics: Conference Series, 012150 doi:10.1088/1742-6596/96/1/012150, vol. 96, (2007).

[14] L. Kocarev and U. Parlitz, "General approach for chaotic synchronization with application to communication”, Phys. Rev. Lett., vol. 74, (1995), pp. 5028-5031.

[15] M. Lin, Z. Yuan and J. Cai, "Finite-time synchronization between two different chaotic systems with uncertainties", arXiv: 0908.0038, (2009).

[16] H. Lin, J. Cai and J. Wang, "Finite-Time Combination-Combination Synchronization for Hyperchaotic Systems", Hindawi Publishing Corporation. Journal of Chaos, vol. 2013, no. 7, (2013).

[17] Y. Liang and J.H. Marquez, "Gain Scheduling Synchronization Method for Quadratic Chaotic Systems", IEEE Transactions on circuits and systems-I, vol. 55, no. 4, (2008), pp. 1097-1107.

[18] H. B. Manfeng and Z. Xua, “Adaptive feedback controller for projective synchronization”, Nonlinear Analysis: Real World Applications, vol. 9, (2008), pp. 1253-1260.

[19] K. Murali and Lakshmanan, "Secure communication using a compound signal from generalized synchronizable chaotic systems", Phys. Lett. A, 241(6), (1998), pp. 303-310.

[20] B. Nana, P. Woafo and S. Domngang, "Chaotic synchronization with experimental application to secure communications", Communications in Nonlinear Science and Numerical Simulation, vol. 14, no. 5, pp. 2266-2276, (2009)

[21] M. J. Ogorzalek, “Taming chaos - Part I: Synchronization”, IEEE Trans. Circuits and Systems, vol. I 40, no. 10, (1993), pp. 693-699.

[22] F. Pasemann, "Synchronized chaos and other coherent states for two coupled neurons", Physica D, vol. 128, (1995), pp. 236-249

[23] J. H. Park, S. M. Lee and O. M. Kwon, "Adaptive synchronization of Genesio-Tesi chaotic system via a novel feedback control”, Physics Letters A., vol. 371, (2007), pp. 263-270.

[24] J. H. Park and O. M. Kwon, "A novel criterion for delayed feedback control of time-delay chaotic systems", Chaos, Solitons and Fractals, vol. 17, (2003), pp. 709-716.

[25] L. M. Pecora and T. L. Carroll, "Synchronization in chaotic systems", Phys. Rev. Lett., vol. 64, no. 8, (1990), pp. 821-824.

[26] S. Rasappan and S. Vaidyanathan, "Hybrid Synchronization of n-scoll Chaotic Chua Circuits using Adaptive Backstepping Control Design with Recursive Feedback", Malaysian Journal of Mathematical Sciences, vol. 7, no. , (2013)2, pp. 2019-2046.

[27] A. Rodriguez-Angenles and H. Nijmeijer, "Mutual synchronization of robots via estimated state feedback: A cooperative approach", IEEE Trans. Control System Technology, vol. 12, (2004), pp. 542-554.

[28] T. Ushio, "Synthesis of synchronized chaotic systems based on observers", Int. J. Bifurcation and Chaos, vol. 9, no. 3, (1999), pp. 541-546.

[29] U.E. Vincent and R. Guo, "Finite-time synchronization for a class of chaotic and hyperchaotic systems via adaptive feedback controller", Physics Letters, vol. 375, no. 24, (2011), pp. 2322-2326.

[30] S. Vaidyanathan, S. Sampath, "Sliding Mode Controller Design for the Global Chaos Synchronization of Coullet Systems", Advances in Computer Science and Information Technology. Networks and Communications, vol. 84, no. (2012), (2012), pp. 103-110.

[31] X.F. Wang and G. Chen, "Synchronization in Scale-Free Dynamical Networks: Robustness and Fragility", IEEE Trans. Circ. and Syst., vol. 49, (2002), pp. 54-62.

[32] C. W. Wu and L. O. Chua, "A unified framework for synchronization and control of dynamical systems", Int. J. Bifurcation and Chaos, vol. 4, no. 4, (1994), pp. 979-998.

[33] Y. Wang, Z.H. Guan and H.O. Wang, "Feedback and adaptive control for the synchronization of Chen system via a single variable”, Phys. Lett. A, vol. 31, no. 2, (2003), pp. 34-40.

[34] C. W. Wu and L. O. Chua, "A simple way to synchronize chaotic systems with applications to secure communication systems", Int. J. Bifurcation and Chaos, vol. 3, no. 6, (1993), pp. 1619-1627.

[35] S. Xuerong and W. Zhongxian, "Adaptive Synchronization of Coullet Systems with Mismatched Parameters Based on Feedback Controllers", International Journal of Nonlinear Science, vol. 8, no. 2, (2009), pp. 201-205. 
[36] C. C. Yang, "Adaptive control and synchronization of identical new chaotic flows with unknown parameters via single input", Applied Mathematics and Computation, vol. 216, (2010), pp. 1316-1324.

[37] H. T. Yau, "Design of adaptive sliding mode controller for chaos synchronization with uncertainties", Chaos, Solitons and Fractals, vol. 22, (2004), pp. 341-347.

[38] W. Yang, X. Xia, Y. Dong and S. Zheng, "Finite Time Synchronization between Two Different Chaotic Systems with Uncertain Parameters", Computer and Information Science, vol. 3, no. 3, (2010), pp. 174 179.

[39] M. Zochowski, "Intermittent dynamical control”, In Physica, vol. 145, (2000), pp. 181-190.

[40] G. Cai, Z. Tan, "Chaos Synchronization of a New Chaotic System via Nonlinear Control", Journal of Uncertain Systems, vol. 1, no. 3, (2007), pp. 235-240.

[41] B. H. Jian, H. Yan and D. Z. Ling, "Synchronization in the Genesio-Tesi and Coullet systems using the backstepping approach", J. Phys.: Conf. Ser., vol. 96, no. 96, (2008), pp. 1-6. 